\title{
Direction Tuning of Individual Retinal Inputs to the Turtle Accessory Optic System
}

\author{
Naoki Kogo, ${ }^{1}$ Doris McGartland Rubio, ${ }^{2}$ and Michael Ariel, ${ }^{1}$ \\ Departments of ${ }^{1}$ Anatomy and Neurobiology and ${ }^{2}$ Research Methodology, Saint Louis University, \\ Saint Louis, Missouri 63104
}

Neurons in turtle accessory optic system [basal optic nucleus $(B O N)]$ were recorded to study convergence of retinal afferents, using whole-cell patch electrodes in a reduced in vitro brainstem preparation with the eyes attached. BON cells primarily exhibit EPSPs from a contralateral retinal ganglion cell input and generate an output of action potentials. Visual responses were evoked by different directions of either full-field or local moving patterns. Direction tuning of action potentials was compared with that of EPSPs detected by passing the membrane voltage through an AC amplifier and window discriminator. This rough measure of retinal input indicated that the direction tuning of the full-field excitatory input from the retina matched that of the spike output for the same BON cell.

Using local patterns within the receptive fields of the BON cells, it was estimated that one to four adjacent retinal inputs were being stimulated. The direction tuning of these inputs had preferred directions that were similar to that of the full-field spike output of the cell, irrespective of where the small window was placed within the receptive field. Because more than one retinal input may have been stimulated by the small stimulus window, subsets of those EPSPs that may represent responses of a single retinal afferent were identified based on their amplitude and rise time. Again, the preferred direction of those putative single retinal afferents matched the direction tuning of the spike output of the BON cell. These findings are discussed in terms of the formation of the retinal slip signal by the BON.

Key words: EPSPs; basal optic nucleus; brainstem; synaptic convergence; direction sensitivity; retinal slip
In vertebrate sensory systems, it is difficult to investigate information processing at the level of the role of individual synaptic inputs to a single neuron. Most sensory stimuli activate several sensory afferents with overlapping receptive fields that then synapse on the same second-order brainstem neuron. This study used the accessory optic system, the inputs of which are sets of widely distributed, direction-sensitive (DS) retinal ganglion cells. The synaptic convergence of the retinal afferents enable local retinal signals to be converted into a spike code of the retinal slip signal used for oculomotor control (Soodak and Simpson, 1988).

Different sets of DS ganglion cells are known to tile the retina so that their large dendritic trees do not arborize in the territory of any adjacent DS cell from its own set. Such sets have been distinguished based on either their anatomical coupling, revealed by intracellular injections of neurobiotin (Vaney, 1994), or their preferred direction to moving spots of light (Amthor and Oyster, 1995). DS cells of the turtle accessory optic system [e.g., basal optic nucleus (BON)] may analyze a visual scene by summing DS retinal inputs, the preferred directions of which are normally distributed around a mean direction (A. Rosenberg and M. Ariel, unpublished observations). The experiments described below directly measure the convergence of retinal inputs onto a single BON neuron to determine whether the preferred direction of its inputs match the preferred direction of its spike output. One possibility is that the cell receives retinal inputs that are all

\footnotetext{
Received July 21, 1997; revised Jan. 9, 1998; accepted Jan. 9, 1998.

This work was supported by Grant NS33190 to M.A. We thank Dr. Alex Rosenberg for comments on this manuscript.

Correspondence should be addressed to Dr. M. Ariel, Department of Anatomy and Neurobiology, Saint Louis University, 1402 South Grand Boulevard, St. Louis, MO 63104.

Copyright (ㄷ) 1998 Society for Neuroscience $\quad 0270-6474 / 98 / 182673-12 \$ 05.00 / 0$
}

excitatory from a single set of DS ganglion cells. If that set is made up of cells that prefer a common direction of retinal image motion, then their synaptic convergence would serve to spatially integrate those inputs to enlarge the receptive field.

Another possibility for synaptic integration is that different sets of DS retinal cells synapse directly on the same brainstem neuron, some that excite in the preferred direction and others that inhibit in the opposite direction. This redundant sensory information results in a more robust sensory signal. A third scheme is that the accessory optic system receives inputs from different sets of DS cells that prefer different directions of motion that vary locally across its receptive field (Burns and Wallman, 1981; Simpson et al., 1988; Wylie and Frost, 1990; Krapp and Hengstenberg, 1996).

In this study, we begin the analysis of the visual inputs to the accessory optic system on a cellular level by using a reduced turtle brain preparation so that the synaptic events are only from retinal inputs to the BON and any intra-BON synapses but not from other brain regions. In this way, the direct convergence of retinal input to the BON is first analyzed to identify whether the inputs are DS and, if so, what their distribution of preferred directions is. It is known that at least some of the direct input to BON is from DS cells (Rosenberg and Ariel, 1991), as shown by recording from DS retinal ganglion cells and antidromically stimulating them by microstimulation of the contralateral BON. Those extracellular experiments could not reveal the functional neural circuitry from the retina to the BON; e.g., does a nasal-preferring BON cell receive synaptic inputs from, and only from, nasalpreferring retinal afferents across its receptive field? However, whole-cell patch recordings from a BON cell provide sufficient resolution to record EPSPs from many retinal afferents and to distinguish individual afferents based on their shape (Kogo and 
Ariel, 1997). Our findings support the hypothesis that BON cells receive inputs from $D S$ retinal ganglion cells, the preferred directions of which are similar. The neural convergence in the BON may be the basis for the conversion from local retinal directional information into a measure of global image motion, retinal slip, that has a well-defined role in vestibular and oculomotor reflexes.

\section{MATERIALS AND METHODS}

The animal care and experimental preparation are described in detail elsewhere (Rosenberg and Ariel, 1990; Kogo and Ariel, 1997). Turtles, Pseudemys Scripta elegans, were maintained in a room temperature aquarium before the $>1 \mathrm{hr}$ of cryanesthesia in ice water. The entire brain was removed with the two eyes attached. The eyes were hemisected so that visual stimuli could be focused onto each retina. To achieve a sheet-like structure in an interface chamber, many dorsal brain structures were removed; i.e., telencephalon, dorsal thalamus, pretectum, tectum, and cerebellum. This ablation also removed indirect retinal $\rightarrow$ BON pathways that are a source of IPSPs (Kogo and Ariel, 1996).

The superfusate (in mm: $\mathrm{Na} 130, \mathrm{~K} 2.0, \mathrm{Ca} 3.0, \mathrm{Mg} 2.0, \mathrm{Cl} 97$ ) was bubbled with $95 \% \mathrm{O}_{2}-5 \% \mathrm{CO}_{2}$ gas so that the $\mathrm{pH}$ of the solution was $\sim 7.6 \pm 0.05$ and its osmolarity was $\sim 274 \pm 2 \mathrm{mOsm}$. Glass micropipettes (5-9 $\mathrm{M} \Omega$ ) were filled with another solution (in mM: $\mathrm{KMeSO}_{4} 124, \mathrm{CaCl}_{2}$ 2.3, $\mathrm{MgCl}_{2}$ 1.2, HEPES 10.0, EGTA 5.0, ATP 2.0; pH 7.3-7.4; osmolarity, $264 \mathrm{mOsm})$.

Visual stimulation. Details of visual stimulation in this in vitro preparation can be found elsewhere (Amamoto and Ariel, 1993). In a darkened room, a full-field stimulus was generated on a computer monitor and focused through a lens to cover the whole retinal eyecup contralateral to the recording. Stimuli were moved in either 12 or 18 different directions interrupted by a $1 \mathrm{sec}$ pause. From the geometry of the $640 \times 480$ pixel monochrome monitor positioned above this retina, a video pixel equaled $11 \mu \mathrm{m}$ on the retina or $\sim 0.13^{\circ}\left(8.25^{\prime}\right)$ of visual angle (Northmore and Granda, 1991).

Based on a preferred direction that was determined during the fullfield stimulation, the receptive field was mapped using a spot moving in the preferred direction. Then, within that receptive field, the local moving pattern was composed of equally spaced squares or spots that gradually disappeared at the edge of a stimulation window as another element reappeared on the opposite edge of the stimulation window. In this way, both the direction of stimulus motion and the total luminance on the retina remained constant. As with any type of local moving patterns, a retinal ganglion cell, the receptive field of which lies at the edge of the stimulation window, may also respond to stimulus onset or offset as a moving element appears or disappears, albeit gradually. This local motion stimulus was generated either by software or simply by masking a full-field pattern with an opaque film from which a round window was cut. The responses to these two local patterns were similar.

Data recording and on-line analyses. During visual stimulation, the membrane voltage was stored on videotape after digitization at a $44 \mathrm{kHz}$ sampling rate. Also, EPSPs were detected by filtering the membrane voltage through an AC amplifier ( $3 \mathrm{~dB}$ cutoff from 500 to $20,000 \mathrm{~Hz}$ ) and sent to a window discriminator that produced $100 \mu \mathrm{sec} 5 \mathrm{~V}$ pulses for the computer. The threshold of the discriminator was set to record either spike events (excluding EPSPs below the window) or EPSPs (excluding the smaller transient voltage changes considered to be recording noise) (Kogo and Ariel, 1997). Using this event detection method, hereafter referred to as AC window detection, event times were sampled by the computer at a rate of $250 \mu \mathrm{sec}$ for both EPSP and spike detection using the same stimulus conditions and data collection software (Fan et al., 1993). The computer saved the time of occurrence of each event relative to the stimulus condition. Stimuli of back and forth motions along a given directional axis were interleaved with the other axes across several trials.

EPSPs exhibited some temporal summation during stimuli moving in a preferred direction. Although the duration of EPSPs may be tens of milliseconds, its rising phase was very rapid, and thus the AC-filtered signal was dominated by positive and transient EPSP onset events. Because the EPSPs were quite large relative to the recording noise, high rates of EPSPs could be easily and accurately measured from the filtered signal. Of course, using this method, the rare occurrence of two nearly simultaneous EPSPs could pass through the window discriminator and be counted as one event. However, the likelihood of such EPSP undercounting was so low that it would not affect the estimate of the preferred direction of the synaptic input.

The AC window detection technique was also subject to contamination by IPSPs when the BON cell membrane was hyperpolarized to $-90 \mathrm{mV}$, thus inverting IPSPs into depolarizing events $\left(E_{\mathrm{Cl}^{-}}=-68 \mathrm{mV}\right)(\mathrm{Kogo}$ and Ariel, 1997). However, in BON cells recorded in this brainstem preparation without the dorsal brain, very few IPSPs were observed at $-60 \mathrm{mV}$ with or without visual stimulation. Furthermore, the direction tuning of BON cells using the $\mathrm{AC}$ window detection technique was similar to the results of the laborious off-line analysis in which EPSPs were individually viewed and measured. Therefore, AC window detection was considered a quick and reliable method to evaluate the visual response properties of the excitatory synaptic events.

Off-line analyses. Kogo and Ariel (1997) reported that unitary EPSPs could be evoked in a given BON cell by retinal microstimulation within that receptive field of the cell. The shapes of unitary EPSPs, measured as amplitudes and rise times, were often very similar for the same afferent but different from afferent to afferent. Unlike this retinal microstimulation, recordings during visual stimulation evoked several EPSP shapes simultaneously, even during stimulation of a small retinal area. From this variety of EPSP shapes, an off-line analysis was performed to extract clusters of common EPSP shapes that may represent the response from a single afferent. From the frequency of those extracted events for each stimulus direction, a preferred direction was estimated.

Synaptic activity was recorded onto video tape for subsequent analysis using MINI software (kindly provided by Dr. J. H. Steinbach, Washington University, St. Louis, MO) to quantify the shape of each selected synaptic event. The amplitude and rise time of each EPSP were measured by an observer who was naive of the stimulus condition. Then, on scatterplots of these two parameters, apparent clusters of these synaptic events were identified. Boundaries around those regions were defined subjectively using the scatterplot derived from the most responsive condition. Finally, direction tuning of events within those boundaries was analyzed from scatterplots derived from the different stimulus directions.

In general, there were few events elicited during pattern movement within a small window. Therefore, to determine objective boundaries of regions of the scatterplots, cluster analysis was performed on data pooled from several presentations of one or several preferred directions, as follows. Data sets recorded during preferred direction stimulation were subjected to additional processing, because these data sets had the largest number of events to subdivide into clusters. All parameters were standardized to a mean of 0 and SD of 1 before analysis. First, the distance between each possible pair of data points in the data set (squared Euclidean distance) was measured based on the two parameters (amplitude and rise time). Then, the mean of the distances from each data point and its 10 nearest neighbors was used as the density measure for a cluster analysis of the three parameters: rise time, amplitude, and density (K-means clustering, SPSS). The number of legitimate clusters to extract from each data set was determined empirically by testing different possible cluster numbers to find the largest number of clusters for which each cluster still had a relatively large number of elements. For these data sets, the optimal cluster number was either two or three. Then, using that cluster number, a two-parameter cluster analysis (amplitude and rise time) was performed on the same data set to determine clusters (by computer) and to draw boundaries between them (by hand). Compared with the subjective clusters observed visually, the objective boundaries were broader, because the software did not exclude outliers. The objective boundaries were then applied to the remaining data sets recorded during stationary patterns or nonpreferred motion. The number of events within these clusters was plotted in polar coordinates as a function of stimulus direction to display the direction tuning of synaptic events that have those objectively determined shapes.

The clusters of synaptic events on scatterplots were also compared with 25 published sets of synaptic events that actually represent responses to microstimulation of single retinal ganglion cell afferents to a given BON cell (Kogo and Ariel, 1997, their Fig. 11). In that study, minimal stimulation was used to evoke unitary EPSPs just above the threshold for bipolar retinal microstimulation within the BON cell receptive field in the contralateral eyecup. To make a statistical comparison with the objectively defined clusters, those 25 data sets were processed to remove stimulation failures and outliers. The membrane voltage after a stimulation was considered a failure when it fell below the nonsynaptic noise of that recording. Values were outliers if they were $>2$ SD from the mean of the amplitude or rise time. A multivariate ANOVA was performed using amplitude and rise time as the dependent variables within each of the data sets as the factors (criterion of $p<0.05$ ). The result of that analysis was a percentile value for each data set, indicating how many of the other sets were statistically different from that data set (e.g., $66.7 \%$ 
indicates that the data set was different from two of three of the other sets; $100 \%$ indicates that the data set was different from all the other sets).

The direction-tuning curves were quantified using two threeparameter fitting methods: the rectified sinusoid equation (Rosenberg and Ariel, 1991) and the wrapped normal equation. Both fitting methods objectively estimated the preferred direction from the phase parameter. The rectified sinusoid equation computed offset and modulation depth parameters (Rosenberg and Ariel, 1991), whereas the wrapped normal equation computed variance and a scaling factor. Fits to the rectified sinusoid equation did not produce a measure of directional tuning width. Also, their correlation coefficient values were reduced for rectified data, unless response values near zero were excluded to allow for negative fit values. Fits to the wrapped normal equation did not suffer these problems.

Rosenberg and Ariel (1991) have reported that responses of DS retinal ganglion cells and BON cells are DS for most of the stimulus axes. This breadth in direction-tuning curves makes accurate estimates of preferred direction difficult. During pattern movement within a small window, only a few events were sometimes elicited in each cluster, thus making the estimates of their preferred directions less accurate. These estimates were rejected if their correlation coefficients fell below 0.6, the criterion value for DS responses (Rosenberg and Ariel, 1991). The direction tuning of the spike response of each cell was also compared with that of published extracellular BON spike data (Fan et al., 1995). For this latter analysis of those two samples, one cell from the extracellular sample and three cells from the intracellular sample were excluded for failing the DS criterion.

\section{RESULTS}

Visual response properties were measured for both the spike output of the BON cell and the EPSP input to the BON cell. The first analysis compared the spike output measured in these wholecell recording experiments with those published reports using extracellular electrodes. Typical responses from whole-cell and extracellular recordings demonstrate that both techniques provide identical measures of direction-tuning properties (Fig. 1, compare $A$, extracellular responses recorded with a varnished tungsten electrode, and $B$, intracellular recordings as described above). The similarity exists, although the whole-cell recording method had the following differences relative to previous extracellular experiments: (1) action potentials had a much larger signal-to-noise ratio; (2) the BON cells were encountered by the physical proximity to the patch electrode tip and not by using a visual search stimulus to isolate only a visual responsive unit; (3) the ruptured BON cell had its internal cytoplasmic contents dialyzed during whole-cell recording, which could change its physiological properties; and (4) the brainstem preparation was missing much of the dorsal brainstem.

The entire samples of BON recordings (extracellular, $n=194$; intracellular, $n=99$ ) were also compared based on the fits of direction-tuning curves (see Rosenberg and Ariel, 1991). Although the sample size differed, the preferred directions of each sample were similarly distributed (Fig. 1, insets). The mean spontaneous activity during whole-cell recordings was quite variable, $1.84 \pm 2.34(\mathrm{SD})$ spikes/sec and was thus not significantly different ( $t$ test at the 5\% level) from that of the extracellular recordings $(0.51 \pm 0.08 \mathrm{spike} / \mathrm{sec})$. From the distribution of the preferred directions, it is clear that the likelihood of encountering BON cells of specific preferred directions is also similar for the wholecell and extracellular techniques. However, these analyses confirmed that, despite the invasive aspect of rupturing a patch of BON cell membrane, visual responses using that approach were roughly equivalent to spike activity of extracellular unit recordings. It is likely therefore that the BON contains a nonuniform distribution of preferred directions. The paucity of BON cells preferring nasal motion is not simply an artifact of an extracel-


Figure 1. Extracellular versus whole-cell patch recordings from the BON. Direction tuning of visual responses was recorded by each technique using an identical full-field stimulus generated on the computer monitor. Both sets of data show direction tuning in response to 18 stimulus directions. The polar positions for the two cells were rotated to align their preferred directions. $A$, Extracellular data are displayed as 5 sec peristimulus spike histograms that include a preceding $0.8 \mathrm{sec}$ period before stimulus motion onset. $B$, Intracellular data are displayed as individual $1 \mathrm{sec}$ voltage traces during a single stimulus presentation. At the fast time scale of these traces, the response latency inherent in retinal processing and conduction to the $\mathrm{BON}$ are evident. Below each trace is a horizontal $-80 \mathrm{mV}$ reference line. Within each panel are polar plots derived from responses averaged from the total stimulus duration for each direction (18 sec extracellular, $12 \mathrm{sec}$ intracellular). These graphs plot the event frequency as the length from the origin and the stimulus direction as the plot angle relative to nasal visual field motion (to the right). In addition to the data points, a curved line shows a computer-generated fit, from which the preferred direction was measured. Insets below each polar plot, Distributions of preferred directions of each sample, both as a single line for each cell and as a polar histogram of $20^{\circ}$ bins. 
Figure 2. Direction-tuning curves of spikes and EPSPs during hyperpolarizing current injection. Curves are displayed in cartesian coordinates for the 12 stimulus directions of a full-field pattern (abscissa, stimulus direction; ordinate, event frequency; triangles, spikes; filled circles, EPSPs). The curved lines represent the computer fit to the data points; the horizontal lines represent the levels of spontaneous activity measured during stationary periods of the visual pattern. Below each curve are symbols to show the preferred directions of each wrapped normal fit. $A, B$, top row, Two cells before and during membrane hyperpolarization. Activity was averaged across the total stimulus duration of 15 $\mathrm{sec}$ for each direction. The cell membrane was either at rest $(-60 \mathrm{mV}$, filled triangles, solid lines) or hyperpolarized to $-90 \mathrm{mV}$ (open triangles, dotted lines). $C, D$, bottom row, Two different cells and comparison of their spike $(-60 \mathrm{mV}$, filled triangles, solid lines $)$ and EPSP responses $(-90 \mathrm{mV}$, filled circles, dotted lines). The dotted horizontal lines indicate that the level of spontaneous EPSPs was much higher than that of the spontaneous spikes. Note that the preferred direction of the EPSP input was similar to the BON output. In $C$, EPSP modulation occurred on top of the spontaneous EPSP rate, whereas the cell in $D$ showed inhibition of EPSPs during stimulus motion in nonpreferred directions.
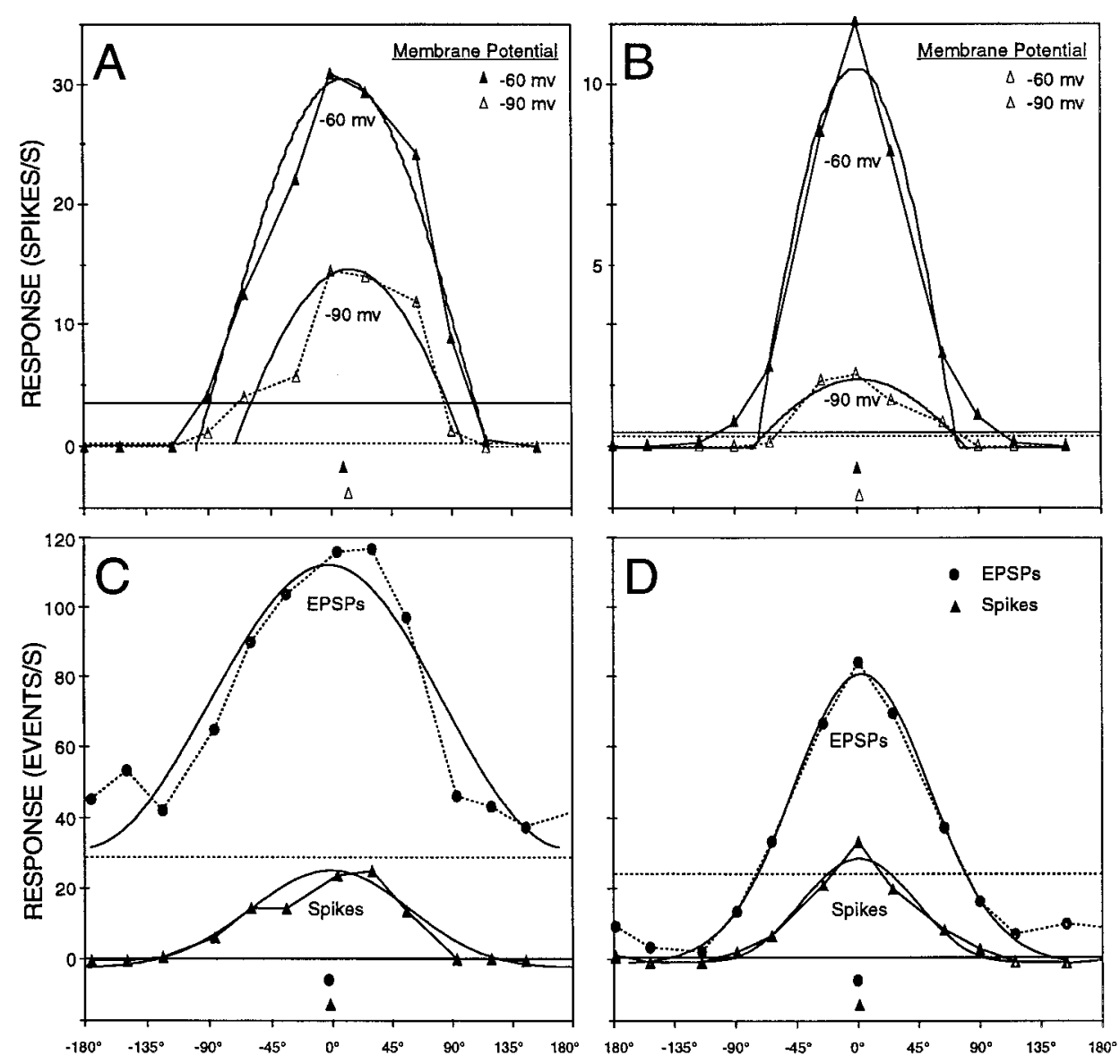

lular electrode bias in an intact brainstem (Fan et al., 1995) or a consequence of the reduced brainstem preparation.

Comparing the two methods of fitting the direction-tuning curves showed that the phase measurement of the wrapped normal fit and sinusoidal fit were within $1.5^{\circ}$ on average $\left( \pm 2.8^{\circ}, n=\right.$ 54 ), indicating that either approach was adequate to provide an objective measure of the preferred direction of each cell. We also found here that, like the extracellular data analysis, directiontuning curves of visual responses during whole-cell recordings were also better fit by a wrapped normal equation than by a rectified sinusoid equation (mean correlation coefficients $=0.93$ and 0.74 , respectively), although both methods were threeparameter fits. When response values near zero were excluded to allow for negative fit values, the average correlation coefficient of the sinusoidal fit increased to 0.89 . Because a wrapped normal equation accommodated direction-tuning curves better, it appears that the DS inputs to BON cells may be normally distributed around a mean preferred direction to create the DS BON cell spike output (Rosenberg and Ariel, unpublished observations). The remainder of these results will describe experiments to determine the range of preferred direction inputs to a $\mathrm{BON}$ cell and thus deal with whole-cell recordings exclusively.

\section{Effect of current injection through the patch pipette}

It has been shown that the frequency of the spike firing of turtle BON cells is linearly correlated with the amplitude of an injected current (Kogo and Ariel, 1997). Assuming that the synaptic input to each BON cell is the sole determinant of the direction tuning of its spike output, then the preferred direction should be independent of an imposed change in the membrane potential. This was analyzed by testing visual responses before and during hyperpolarizing current injection through the patch pipette. Examples are shown in Figure 2, $A$ and $B$. The effect of hyperpolarizing BON cells from the resting membrane potential of $-60 \mathrm{mV}$ to $-90 \mathrm{mV}$ is shown in the top traces. Because the membrane potential of the cell was relatively further from the threshold voltage for spike initiation, the number of spikes during visual stimulation was reduced, often to zero. In those cases in which spike responses to stimulus motion still occurred, the direction tuning (preferred direction and variance of the wrapped normal fit) remained similar during hyperpolarization (Fig. $2 A, B,-90$ $\mathrm{mV}$, open triangles, dotted line) compared with the cell at rest (Fig. $2 A, B,-60 \mathrm{mV}$, filled triangles, solid line). The difference between the preferred direction at -90 and $-60 \mathrm{mV}$ for our sample was $8.6 \pm 9.0^{\circ}(n=12)$. This result serves as a control for the subsequent analyses in which EPSP recordings were performed at $-90 \mathrm{mV}$.

\section{Visual response properties of all EPSPs occurring in a BON cell during full-field retinal stimulation}

The major excitatory input to the BON comes from retinal ganglion cells. To roughly evaluate the direction tuning of this input, BON cells were hyperpolarized to $-90 \mathrm{mV}$ to reduce spike activity, and then the membrane voltage signal from the DC preamplifier was passed through an AC amplifier. The resulting signal contained transients that represented the occurrences of EPSPs that could be detected as individual events by the window discriminator.

The direction tuning of EPSP inputs to BON cells was found to be well-matched to its spike output (two examples in Fig. 2, 
bottom). In most cases, the data collection occurred during the experiment with the synaptic and spike responses measured within minutes of each other. The simple result was that the average retinal input to a BON cell, an aggregate of EPSP responses, had the same direction tuning during drifting visual patterns as that of the BON spike output (Fig. 2, filled circles vs filled triangles). A similar analysis was performed in another three cells. The mean difference between the preferred direction of EPSPs and spikes for the same cell set to $-90 \mathrm{mV}$ was $16.0 \pm$ $14.7^{\circ}(n=5)$. Because few spikes occurred at $-90 \mathrm{mV}$, this measurement was performed also at $-60 \mathrm{mV}$. Still, the difference between preferred directions of EPSPs $(-90 \mathrm{mV})$ and spikes $(-60 \mathrm{mV})$ for our sample was $15.7 \pm 19.5^{\circ}(n=17)$. This result remains clear, although some EPSPs may have not been counted because of their small size or simultaneous occurrence with another EPSP.

The modulation of the visual EPSP response was superimposed on a high level of spontaneous EPSP events (Fig. 2, dashed lines at bottom). These levels were measured during periods of a stationary pattern that were interleaved among the stimulus trials. Of course, the measurement of spontaneous events depended in part on where the threshold of the window discriminator was set for each cell and on the filter settings of the recording amplifiers (fixed at 500-20,000 Hz). Apart from that absolute level, part of the high spontaneous EPSP rate of some cells was reduced during motion in the nonpreferred direction (Fig. 2D). This EPSP reduction presumably indicates inhibition of spontaneous spike activity in the retina, because IPSPs were rarely observed in BON cells in the reduced brainstem preparations (Kogo and Ariel, 1997).

\section{Visual response properties of EPSPs during local retinal stimulation}

The final analysis of the direction tuning of synaptic input to the BON used small moving visual patterns to evaluate the properties of individual retinal afferents to a BON cell. It was necessary to estimate the receptive field size of a typical afferent. First, the average diameter of the receptive fields of BON cells was determined to be $31.8 \pm 8.1^{\circ}(n=18)$ based on EPSP responses during whole-cell recordings. Based on the topography of backfilled retinal ganglion cells from the contralateral BON (Zhang and Eldred, 1994), one can estimate that the average BON receptive field size can receive a possible input from $109.3 \pm 15.0(n=4)$ ganglion cells (Fig. $3 A$ and as described in Discussion). However, assuming between three and nine subtypes of ganglion cell inputs, each preferring a different direction of motion, it was estimated that only 12-36 retinal ganglion cells might contribute to the visual response of a single BON cell, each with nonoverlapping receptive fields with diameters of between 5.3 and $9.2^{\circ}$.

These estimates suggest that small drifting patterns $\left(3.4-8.8^{\circ}\right.$ width) may only stimulate one or a few retinal ganglion cell inputs to a given BON cell. Therefore, local retinal stimulation was presented while recording EPSPs from a hyperpolarized BON cell, the membrane voltage of which was AC-filtered and heard through an audio amplifier. This approach allowed for rapid observations of the preferred direction responses to many positions within the receptive fields of dozens of BON cells. We never observed a retinal position that had its preferred response in a direction very different from the spike output of the BON cell. The observation was quantified by detecting EPSPs by the AC window during local retinal stimulation in 11 cells. Figure 3 presents two examples (Fig. $3 B, C$; also Fig. $4 A$ ) and shows the

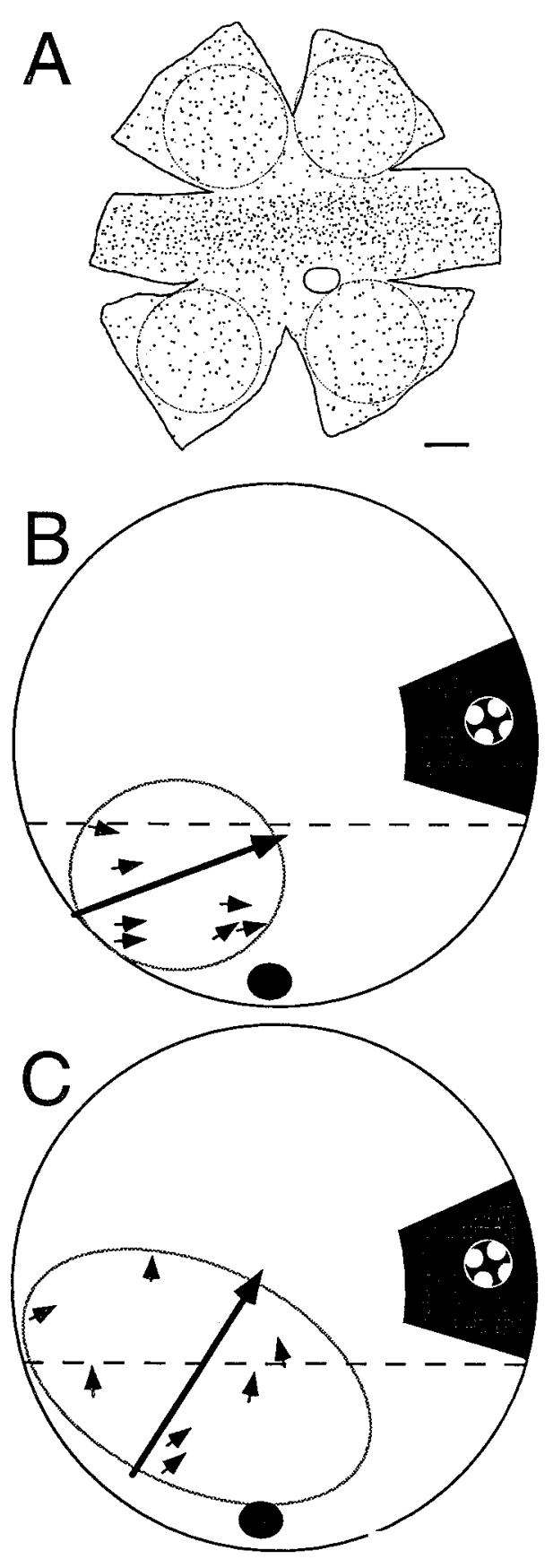

Figure 3. Local retinal ganglion cell input and local visual responses. $A$, Whole-mounted retinal drawing, kindly provided by Dr. William Eldred, (Boston University, Boston, MA) showing all the retinal ganglion cells labeled from a contralateral BON injection of rhodamine. A circle equivalent to the average $\mathrm{BON}$ receptive field $\left(31.8^{\circ}\right)$ was drawn over each peripheral retinal area, and the ganglion cells were counted. Calibration bar, $10^{\circ} . B, C$, Drawing of a right retinal eyecup (outer circle) as viewed from above (nasal retina to the right) shown at twice the scale of $A$. Local stimuli were presented at many stimulation locations within the receptive field (oval) inferior to the visual streak (dashed line) and adjacent to the optic nerve head (black circle). Small arrows are placed on this drawing at the retinal stimulus locations and oriented to point toward the preferred direction of EPSPs recorded in that area as detected by the AC window. The large arrow represents the preferred direction of the BON cell spike output when the entire retina was stimulated. The right inset shows the stimulus as it would appear drawn to scale on the retina in darkness, as a pattern of $2.7^{\circ}$ white spots moved in 1 of 12 directions. The pattern moved within a round stimulus window of $6.9^{\circ}$. 

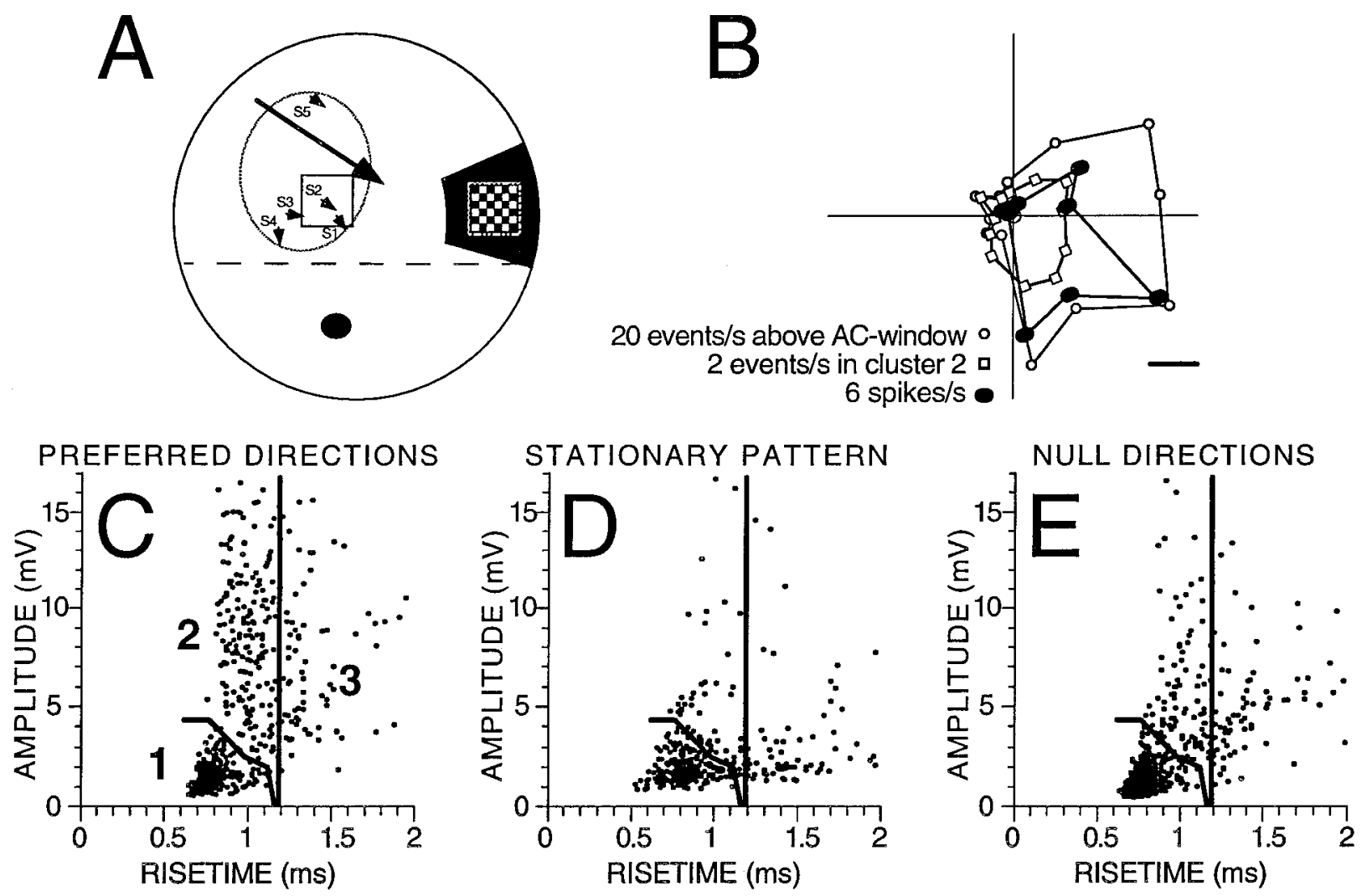

Figure 4. Clustering of EPSP responses based on amplitude and rise time. A, Drawing of a retinal eyecup (as in Fig. 3). Local stimuli were presented at five stimulation locations labeled $S 1-S 5$, and EPSPs were detected by the AC window to estimate the preferred direction of each retinal area. The right inset shows the stimulus pattern of $181.1^{\circ}$ white squares that moved together in 1 of 12 directions within a square stimulus window of $8.8^{\circ}$. $B$, Three superimposed direction-tuning curves plotted as in Figure 1 but rotated to match the orientation of the eyecup shown in $A$. The filled circles show the direction tuning of spikes recorded at $-60 \mathrm{mV}$ during full-field stimulation. The open circles show direction tuning of all EPSPs detected by the AC window at $-90 \mathrm{mV}$ during full-field stimulation. The open squares show direction tuning of cluster $2(C-E)$. The scaling is not the same for the different polar plots but demonstrates that these three response measures of this BON cell preferred similar directions. $C-E$, Amplitude-rise time scatterplots showing the distribution of EPSP shapes recorded in a BON cell hyperpolarized to $-90 \mathrm{mV}$ with the local pattern centered on the $S 2$ stimulation site shown in $A$. Each data point represents the shape of an individual EPSP recorded during equivalent $40 \mathrm{sec}$ periods $(10 \mathrm{sec}$ of local pattern motion in 4 preferred and 4 null direction presentations, $85 \mathrm{sec}$ periods of no motion). From the preferred data in $C$, boundaries were objectively determined to divide the scatterplot into three regions using cluster analysis. Note that there were many more events within the boundaries of cluster 2 in $C$ than were recorded during the null directions in $E$. The stationary pattern $(D)$ evoked the fewest events in all clusters. The direction-tuning curve of cluster 2 is plotted in $B$.

preferred directions of all the tested areas (excluding a few regions with responses that were not DS; i.e., correlation coefficient of the fit $<0.6)$. Across the entire sample, the mean difference between the preferred directions of the DS EPSPs (local stimulation) and spikes (full-field stimulation) was $18.4 \pm$ $11.7^{\circ}(n=31)$. This difference is similar to that reported above $\left(15.7 \pm 19.5^{\circ}, n=17\right)$ comparing EPSPs during full-field stimulation $(-90 \mathrm{mV})$ with spikes during full-field stimulation $(-60$ $\mathrm{mV}$ ). Although the variability of these preferred direction measurements may relate to the estimation procedure, it is clear that the preferred direction of the inputs to BON cells were within $\sim 45^{\circ}$ of the preferred direction of their spike output.

Quantification of unitary EPSP inputs to a BON was much more difficult than the analysis of events detected by the AC window. The unfiltered EPSP events during a local retinal stimulation still had a variety of shapes and sizes, perhaps from input of more than one ganglion cell afferent or from other nonvisual synaptic inputs. An identification of unitary EPSPs from individual ganglion cell inputs was therefore attempted based on our previous finding that different afferents have distinctive and characteristic EPSP amplitudes and rise times (Kogo and Ariel, 1997, their Fig. 11). Five BON cells were selected for this further analysis, because their rates of spontaneous synaptic activity were low, and the responses to local moving patterns had large DS EPSPs.

In the first example, Figure $4 A$ shows the specific retinal subregion that was stimulated to record EPSPs, the amplitudes and rise times of which are plotted in Figure 4, $C$ and $E$. Initially, five positions $(S 1-S 5)$ were stimulated within the receptive field of the BON cell, as described for Figure 3, using a local moving check pattern (Fig. $4 A$, inset). Although the estimates of the preferred direction were less reliable, because fewer synaptic events were evoked by local patterns than full-field stimuli, note that each of the five retinal positions (Fig. $4 A$, small arrows) had preferred directions similar to the spike responses to full-field stimulation (Fig. $4 A$, large arrow). The $S 2$ arrow, which aligned closest to the preferred direction of the spike response (Fig. $4 B$, within $16^{\circ}$ based on the phase of a wrapped normal fit), represents data that had the highest correlation coefficient $(0.97$; S1 and S3-S5 values were <0.9). Amplitude-rise time scatterplots were generated from responses to $\mathrm{S} 2$ for each of 12 stimulus directions (Fig. $4 C, E)$ and compared with scatterplots derived from recordings 
during a stationary pattern (Fig. 4D). As can be seen, EPSPs with faster rise times but larger amplitudes showed a prominent direction sensitivity (Fig. 4, preferred direction, $C$, compared with the null direction, $E$ ). Note that these small regions of EPSPs do not correspond to responses from different retinal positions but are different EPSP shapes evoked by stimulus motion at a single small retinal position.

\section{Cluster analysis}

Although in some regions of some amplitude-rise time scatterplots, clusters of events sharing a common shape were apparent, an objective means was developed to determine boundaries within which to analyze direction tuning (see Materials and Methods). Although these regions varied for each data set, there was always a large and dense cluster of events of small amplitudes and short rise times. This region, referred to as cluster 1, was also apparent in recordings during a stationary pattern (Fig. $4 D$; see Figs. $7 A, 8 A$ ). These motion-insensitive events were found in the same region of the scatterplot as events that were insensitive to retinal lidocaine application, supporting a previous suggestion that small EPSPs may be spontaneous "minis" either from retinal axons or intracranial synaptic inputs (Kogo and Ariel, 1997). Alternatively, these visually insensitive events may result from the spontaneous spike activity of ganglion cells outside of the window of local pattern motion.

Of the EPSP clusters that showed clear direction sensitivity, it was possible that they represent the EPSPs of single DS retinal ganglion cell afferents. A visual comparison of these visually evoked DS EPSP clusters (Fig. 5, V1-V3, equivalent to cluster 2 shown in Figs. 4, 6, 7, respectively) was therefore made with the collection of scatterplots of EPSP shapes derived from unitary retinal ganglion cell afferents that were evoked by electrical stimulation (Fig. 5, E1-E20, derived from Kogo and Ariel, 1997, their Fig. 11). In that study, EPSPs from the same afferent occupied small regions of amplitude-rise time scatterplots, indicating that those unitary EPSPs often had a characteristic shape. It is clear that the visually evoked clusters and the electrically evoked EPSP groups were similar in amplitude and rise time.

EPSP groups evoked by visual or electrical stimulation were next quantified and compared statistically. First, multivariate ANOVA showed that unitary EPSP groups were distinguishable from one another based on either amplitude or rise time (histogram in Fig. 5). In fact, all these groups were statistically different from $>66.7 \%$ of the remaining groups, although individual EPSPs of any one group might not be distinguished statistically from individual EPSPs in other groups based on its shape. Then the three DS EPSP clusters identified in this analysis (Fig. 5, V1-V3) were compared with the groups of unitary EPSPs. These three clusters were statistically distinct from all the electrically evoked EPSP groups and from each other (Fig. 5, hatched bar). Therefore, these visually evoked clusters occupy a region on the scatterplots similar to that of unitary monosynaptic EPSPs from single retinal ganglion cells and yet can also be distinguished from other groups based on their shape parameters.

Cluster analysis was not always able to identify small regions of the scatterplot, as shown in Figure 6 (also see Fig. 5, V2). On the amplitude-rise time scatterplot of the preferred direction responses, we subjectively identified a region of increased density of large synaptic events that were $>20 \mathrm{mV}$. However, cluster analysis did not identify that small cluster, but two other clusters were identified: cluster 1 (small non-DS EPSPs; Fig. 6, inset) and a region much larger than our subjective estimate of events, $>20$
$\mathrm{mV}$. In this case, there may have been two adjacent DS retinal ganglion cell afferents stimulated by the local pattern motion, and those two afferents produced EPSPs with shapes that filled two adjacent regions of the scatterplot. One can imagine a paucity of events in a region dividing cluster 2 in half. However, we used the objective method and performed the direction-tuning analysis on the entire cluster 2 (Fig. $6 B$ ). Surrounding the polar plot are voltage traces during different stimulus directions (as shown in Fig. 1, bottom). A similar analysis was performed for all events $>20 \mathrm{mV}$ (a visually derived subjective criterion; data not shown). That analysis produced a nearly identical direction-tuning curve and a much more limited dispersion of EPSP shapes that compared well with that of E1-E20 (Fig. 5).

The next example shows that the use of amplitude and rise time criteria may be better able to identify individual events as having been derived from a single DS afferent than a direct examination of the voltage traces. The BON cell example of Figure $7 B$ shows $15 \mathrm{sec}$ traces recorded in the preferred and null directions. Under each trace, an arrowhead was placed to denote an EPSP that was contained in cluster 2 , a DS region of the scatterplot (Fig. $7 A$ ). It is important to note that most of the events that occurred during null direction motion (Fig. 7B, top trace) were not part of cluster 2 , although their amplitudes may have been larger than that of cluster 1 . The preferred direction of this cell was estimated by the wrapped normal fit to be $197^{\circ}$ using $\mathrm{AC}$ window detection and $234^{\circ}$ from the objectively identified cluster 2 (Fig. $7 B$, middle plot). These directions are close to that of the estimated preferred direction of $214^{\circ}$ for the spike output of the BON cell during full-field motion at $-60 \mathrm{mV}$.

Figure 8 shows the final example in which cluster 3 , but not cluster 2, was DS. This analysis also demonstrates that amplitude-rise time scatterplots are useful to discriminate clusters of data recorded in the voltage-clamp mode. The pattern in this case was also within the smallest stimulus window tested ( $3.4^{\circ}$ square). Of the three identified clusters (Fig. $8 A$ ), both the low-amplitude, fast rise time cluster and the large-amplitude, fast rise time clusters were not DS (Fig. 8B, 1,2, respectively), although their responses were greater during motion than during the stationary pattern. This indicates that at least some EPSCs were visually driven, although not by a directional input. Another possibility is that the visually driven input was from a DS ganglion cell, yet the local stimulus did not cover its receptive field fully. In that case, the weak non-DS response may be attributable to the appearance and disappearance on a white square and not attributable to its motion across the receptive field of the ganglion cell. The final cluster, however, was clearly DS (Fig. 8B, 3), and its preferred direction aligned well with the spike output of the BON cell (Fig. $8 C)$. These findings indicate that individual afferents to BON cells are DS retinal ganglion cells that prefer similar directions of motion.

\section{DISCUSSION}

Using whole-cell patch recordings of cells in the accessory optic system (AOS) of a reduced in vitro turtle brain, it was shown that sensory afferents provided visual inputs that were excitatory and direction-sensitive and had preferred directions that were similar to the spike output of postsynaptic cells. The substantial retinal convergence of DS cells onto a single AOS cell both enlarged the receptive field and appeared to perform a direct linear transformation of the excitatory synaptic current into the spike responses. These results demonstrate that this first central synapse of the optokinetic reflex arc can create a retinal slip signal from the 

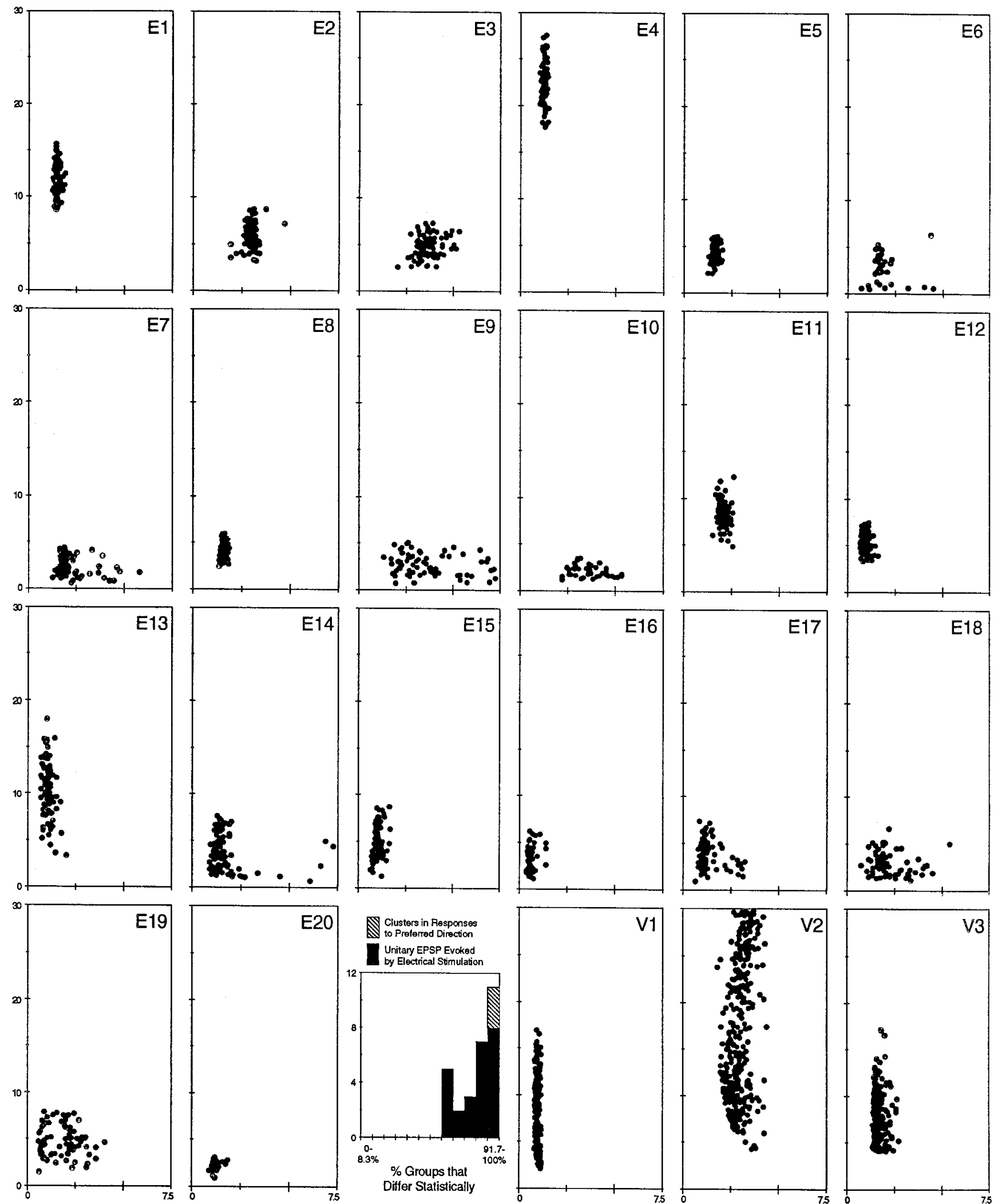

Figure 5. Comparison of visual evoked clusters and electrically evoked unitary EPSPs. Twenty unitary EPSP scatterplots are displayed in the three top rows and left side of the bottom row (E1-E20). V1-V3, Scatterplots of cluster 2 (from Figs. 4, 6, 7, respectively) shown on the right side of the bottom row. These plots all have amplitude in millivolts on the ordinate and rise time in milliseconds on the abscissa. All scatterplots are displayed on the same scale, although the number of points in each plot varied because of electrical stimulation failures caused by near threshold current stimulation or different recording times during visual stimulation. Five unitary EPSP scatterplots, the mean rise time of which was $>5$ msec, are not shown from Kogo and Ariel (1997), their Figure 11. The histogram between E20 and V1 displays the number of EPSP groups as a function of the percentage of remaining groups that were statistically different based on amplitude and/or rise time. 

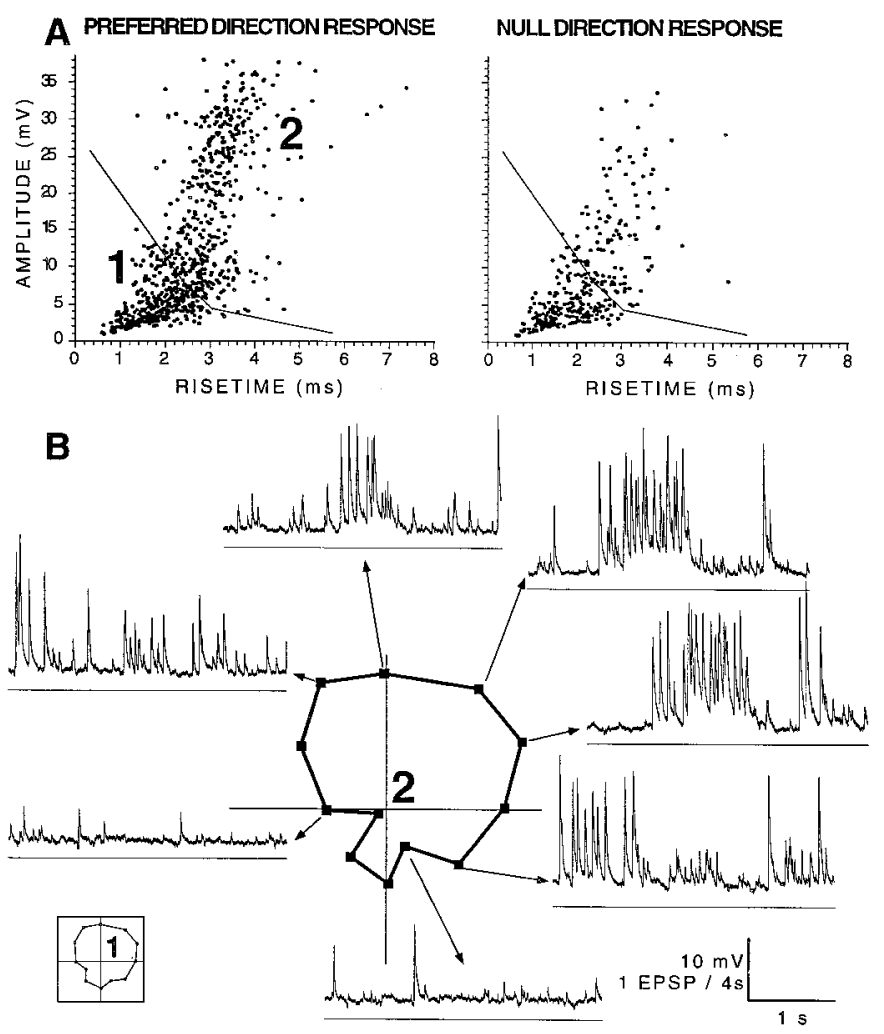

Figure 6. Inability of cluster analysis to divide some large clusters. $A$, Amplitude-rise time scatterplots showing the distribution of EPSP shapes recorded in a BON cell hyperpolarized to $-90 \mathrm{mV}$ during equivalent $40 \mathrm{sec}$ periods ( $8 \mathrm{sec}$ of local pattern motion in 5 preferred and 5 null direction presentations). From the preferred data of the left panel, the boundaries for two clusters were identified objectively. Stimuli and analyses were similar to those of Figure 4, except that the white squares were $2.8^{\circ}$ within a window of only $5.8^{\circ}$ square. $B$, Intracellular data are displayed as individual $2 \mathrm{sec}$ voltage traces for 7 of the 12 stimulus directions. Below each trace is a horizontal $-100 \mathrm{mV}$ reference line. The polar plot in the center represents the direction tuning of the events in cluster 2. Inset to left shows the cluster 1 polar plot.

simple spatial summation of similar DS ganglion cells from across the surface of the retina.

\section{Can the visual response of individual afferent inputs to a single BON cell be studied?}

We have previously shown that an individual retinal afferent to a single BON cell can be stimulated electrically in the eyecup (Kogo and Ariel, 1997). Although a current pulse probably excited more than one cell at the retinal surface, the ability to stimulate a single input to a BON cell electrically may be attributable to a low density of retinal cells that project to any one BON cell. This low density has been shown by backfilling ganglion cells from contralateral BON (Zhang and Eldred, 1994). Of a total of 364,000 ganglion cells (Peterson and Ulinski, 1979), 1500 cells were labeled; each had distinctive dendritic morphology and loop-like patterns that are very similar to physiologically identified DS cells described in rabbit retina (Oyster et al., 1993).

\section{Estimation of receptive field size of single DS afferent}

Without intracellular recordings, it has been difficult to measure the receptive field size of AOS neurons using extracellular techniques. These cells receive a diffuse retinal input that evokes spike activity only when sufficient retinal area is stimulated. A minimum stimulus diameter of $30^{\circ}$ was used by Rosenberg and Ariel (1990) to estimate that the receptive field was $>20^{\circ}$. However, in this study, EPSP responses were readily monitored with an audio monitor for an accurate measurement of the mean receptive field diameter of the $\mathrm{BON}\left(31.8^{\circ}\right)$. Anatomical material of Zhang and Eldred (1994) was then used to estimate the number of inputs to each BON cell. First, in four peripheral regions of the whole-mounted retina that were equivalent to the average BON receptive field (Fig. $3 A, 31.8^{\circ}$ circles), labeled cells were counted to be $109.3 \pm 15$, which we consider the maximum number of retinal inputs to a single BON cell.

Next, we estimated the number of different subgroups found in these 109.3 cells. Bowling (1980) showed three groups of preferred directions in turtle retina. That is a low estimate, because each group is probably subdivided based on its ON-OFF center response. In rabbit, for example, Oyster et al. (1972) described four ON-OFF and three ON-center subtypes of DS ganglion cells. Amthor and Oyster (1995) and Vaney (1994) presented physiological and anatomical evidence that different subtypes of rabbit DS cells tile the retina with a unitary coverage, indicating that the cells of each subtype have dendritic trees that blanket the retina yet do not overlap one another. One would then expect that, for each subgroup, the dendritic spread of individual cells should closely correspond to the distance between neighboring cell somas. Zhang and Eldred (1994) carefully quantified the distance of individual peripheral ganglion cells to their nearest neighboring cell that also projected to the BON as mainly between 25 and $100 \mu \mathrm{m}$. The average distance to a neighboring cell is presumably greater. They also reported the range in the dendritic spread of individual peripheral ganglion cells $(83,000-$ $600,000 \mu \mathrm{m}^{2}$; equivalent to circles of diameter of $\left.325-874 \mu \mathrm{m}\right)$. Presumably, the largest dendritic trees were in the most peripheral retina, where the nearest neighbors are the most separated. In other words, nearly nine of the nearest $100 \mu \mathrm{m}$ neighbors can align within an 874- $\mu$ m-diameter dendritic tree. Assuming unitary tiling, the high estimate for subgroups of DS ganglion cells would be nine for the turtle. This estimate is consistent with subdividing each of the three preferred direction clusters (Bowling, 1980) into subgroups that are ON-center, OFF-center, and ON-OFF type DS cells (Granda and Fulbrook, 1989).

From this estimate of three to nine subtypes of retinal input to BON, then of the 109 potential inputs to each BON cell, only 12 (109/9 subtypes) to 36 (109/3 subtypes) ganglion cells form a single DS retinal subtype that synapses in the receptive field of that BON cell. Furthermore, these 12-36 ganglion cells can be estimated to have nonoverlapping receptive fields of diameters of between 5.3 and $9.2^{\circ}$. This estimate is also consistent with the measured size of the dendritic arbors (area of $83,000 \mu \mathrm{m}^{2}=4.1^{\circ}$ in diameter; area of $600,000 \mu \mathrm{m}^{2}=10.9^{\circ}$ in diameter) (Zhang and Eldred, 1994). With nonoverlapping afferent inputs of those sizes, $3.4-8.8^{\circ}$ visual patterns may have evoked responses from only one or a small number of ganglion cell inputs to a BON cell. This estimate also supports the retinal microstimulation study that showed that closely opposed $\left(\sim 50 \mu \mathrm{m}=0.7^{\circ}\right)$ bipolar electrodes evoked unitary EPSPs to BON cells with low and distinct thresholds (5-200 $\mu \mathrm{A}, 100 \mu \mathrm{sec}$ current pulses) (Kogo and Ariel, 1997).

\section{Examination of the direction tuning of possible single DS afferents}

Even assuming a small number of afferents to a given BON cell, each with nonoverlapping receptive fields, it was necessary to 
A PREFERRED DIRECTION

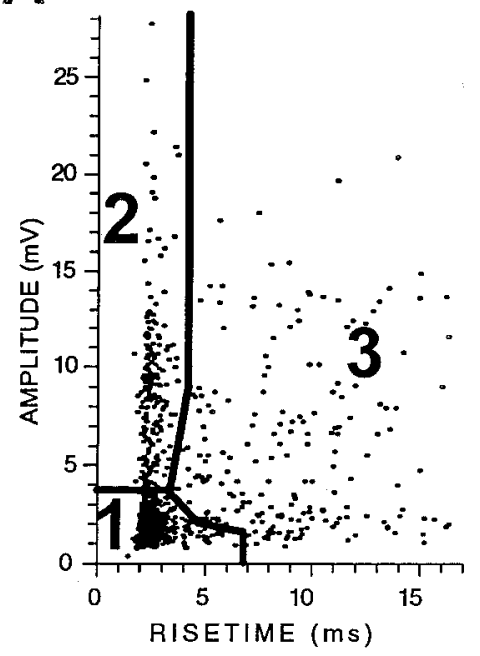

STATIONARY PATTERN

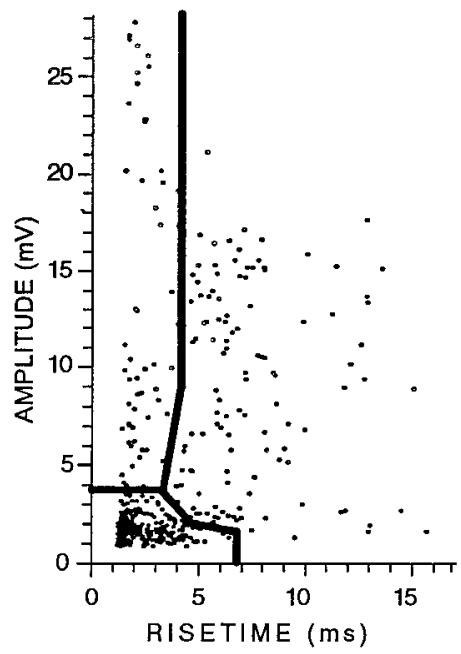

NULL DIRECTION

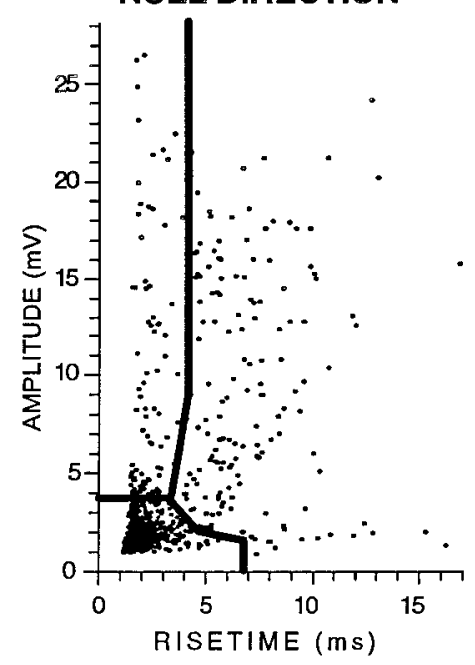

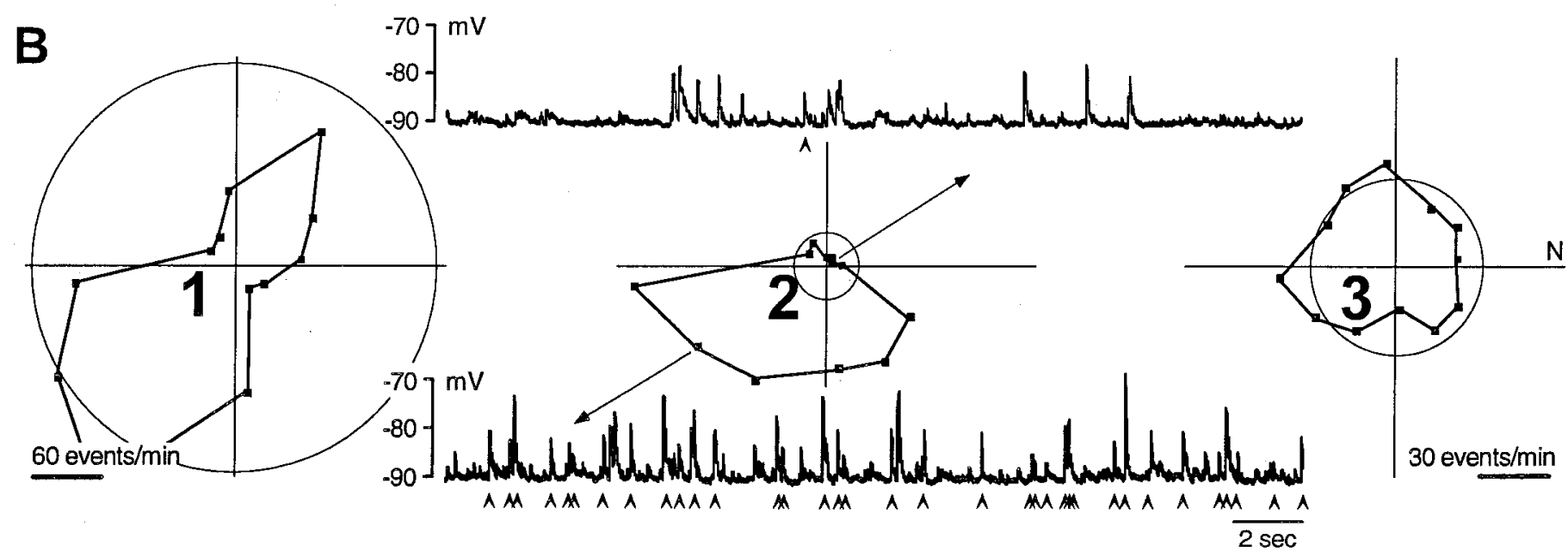

Figure 7. Identification of individual EPSPs from one cluster within a voltage trace. $A$, Amplitude-rise time scatterplots showing the distribution of EPSP shapes recorded in a BON cell hyperpolarized to $-90 \mathrm{mV}$ during equivalent $120 \mathrm{sec}$ periods (two 1 min presentations interleaved for local pattern motion in 12 directions and a stationary condition). From the preferred direction data of the left panel, the boundaries for three clusters were identified objectively. Note that cluster 2 has many more events during the preferred direction than during null direction, except for possible contamination at its lowest boundary. $B$, Three polar plots show the direction tuning of events for each cluster identified in $A$ using the preferred and null direction scatterplots, as well as 10 other scatterplots (data not shown). The circle on each plot shows the spontaneous events derived from the stationary stimulus condition $(A$, middle). Above and below the middle polar plot of cluster 2 are $15 \mathrm{sec}$ voltage traces during null and preferred responses, respectively. Below each trace are arrowheads to identify events, the amplitude and rise time of which fall within the boundaries of cluster 2 . Note that all but one of the EPSPs in this null direction trace had either an amplitude $<3.8 \mathrm{mV}$ or a rise time $>4 \mathrm{msec}$. Note that the calibration of the left polar plot is twice that of the right two plots. There were many more events in cluster 1 than the other two clusters, and those events were inhibited by stimulus motion.

identify which EPSPs were caused by the response of a single afferent from those of other afferents that may also be responding or just spiking spontaneously. Fortunately, EPSPs caused by single retinal afferents have been characterized (Kogo and Ariel, 1997). Retinal microstimulation evoked unitary EPSPs, as judged by finding a clear stimulus threshold and having a fixed stimulus latency. These responses were blocked by retinal application of lidocaine, leaving only small-amplitude events with short rise times. Such lidocaine-insensitive events may be found within the boundaries of cluster 1 of Figures 4 and $6-8$, because events in cluster 1 were invariably non-DS. On the other hand, the largeamplitude unitary EPSPs that were evoked by retinal microstimulation had clear characteristic shapes that separated unitary EPSP groups from one another (Fig. 5).

In this paper, we applied the same approach to EPSPs during movement of a small pattern, although only a subset of them were visually evoked responses. However, the EPSP shape analysis indicated that events of specific ranges of amplitude and rise time may identify visually responsive subsets of EPSPs that were derived from single retinal afferents. Alternatively, the EPSPs from a single retinal afferent are corrupted by a limited number of similarly shaped contaminating EPSPs. Sources for such contamination include (1) a spontaneous retinal ganglion cell from elsewhere in the retina that was not visually evoked from that local retinal stimulation; (2) another retinal ganglion cell, the mean EPSP shape of which was different, but some of its EPSPs were not excluded adequately by the cluster analysis technique (e.g., visual inspection of cluster 2 from responses of both Figs. $6 A, 7 A$ indicate contaminating events, because the lower boundaries appear too low), and (3) the unlikely occurrence that stim- 
A

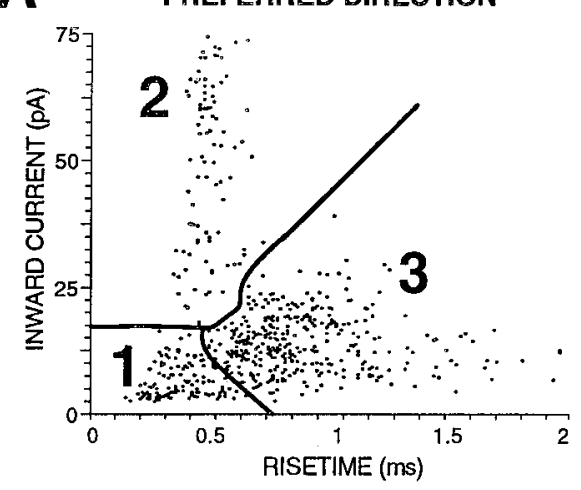

B EPSCs at $-90 \mathrm{mV}$ evoked by LOCAL PATTERN MOTION

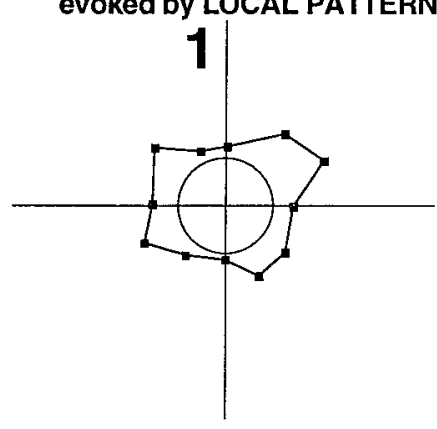

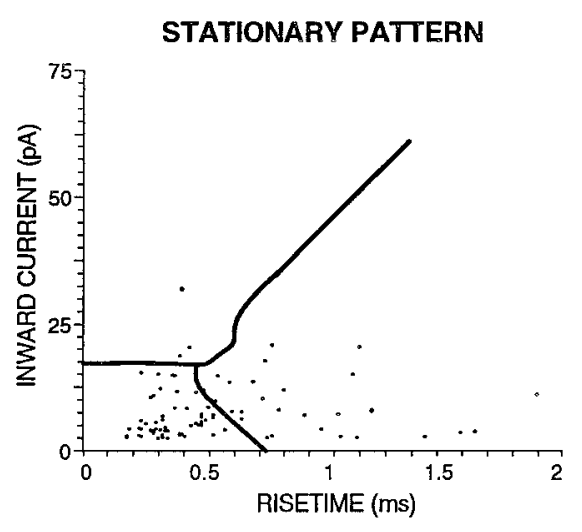

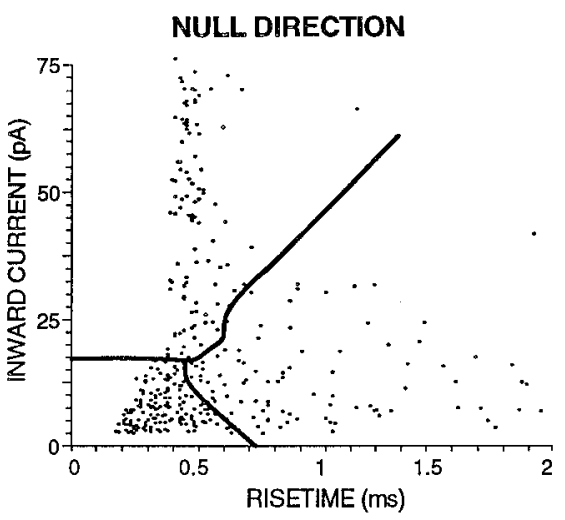

C SPIKES at $-60 \mathrm{mV}$ evoked by FULL-FIELD MOTION

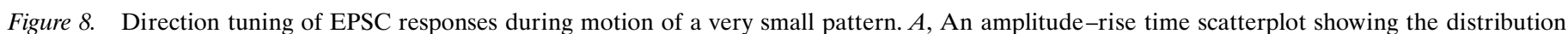

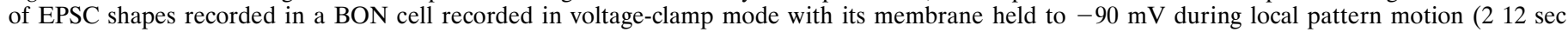

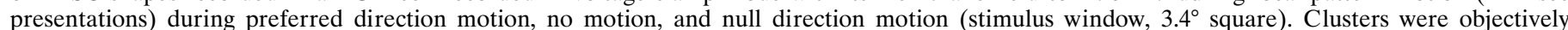

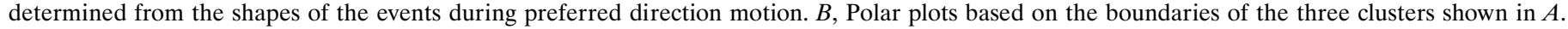

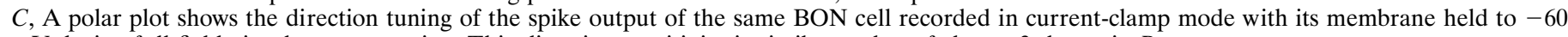
$\mathrm{mV}$ during full-field visual pattern motion. This direction sensitivity is similar to that of cluster 3 shown in $B$.

ulation of one retinal location evoked EPSPs of identical mean shapes from two nearby retinal ganglion cells projecting to the same BON cell. The objective identification of these clusters was difficult, because single afferents may produce too few events among EPSPs that were not visually responsive. Despite these difficulties, small regions of amplitude-rise time scatterplots were found to be DS, with preferred directions similar to that of the spike output of the BON cell.

\section{Complex or simple visual processing in the AOS?}

A simple assumption is that retinal inputs to the brainstem are excitatory and visually tuned to the visual features displayed in the postsynaptic cell. In the lateral geniculate nucleus, the spike output of a cell correlated well with extracellularly recorded EPSPs called S potentials in certain behavioral states (Fourment et al., 1984). The results described here also show that one property of retinal inputs to the AOS, direction tuning, does match the AOS spike output in turtle. DS responses of the AOS of other species may also be attributable to retinal processing, because DS cells are found in most vertebrate species.

Oyster et al. (1972) first suggested that DS ganglion cells played a role in the formation of the retinal slip signal to drive optokinetic eye movements. Simpson et al. (1988) suggested that the AOS relays retinal slip to the cerebellum and vestibular system to serve as an error signal for the control of vestibular ocular reflex gain. In turtles, the properties of BON and DS ganglion cells in vitro have been correlated with the optokinetic behaviors in vivo
(Ariel, 1989, 1990; Rosenberg and Ariel, 1990, 1996). More recently, a model developed by Rosenberg and Ariel suggests that an appropriate variance in a normal distribution of preferred directions of elementary movement detector inputs could account for the direction- and speed-tuning properties measured in the sample of extracellular BON spike responses (Rosenberg and Ariel, unpublished observations).

After convergence of retinal inputs onto an AOS cell, it is still possible that the AOS spike output is normally modified by indirect visual inputs from the contralateral AOS or overlying pretectum, both of which were removed surgically in this reduced preparation. Pretectal neurons also encode retinal slip but prefer different directions of motion (Baldo and Britto, 1990; Natal and Britto, 1987; Fan et al., 1995). Similarly, DS input may come from visual cortex that derives a measure of visual motion direction independent of the retina (Grasse et al., 1984; Hamassaki et al., 1988; Natal and Britto, 1988). The combined output of retinal and cortical directional processing may result in AOS response to a broader range of stimulus velocities, as well as binocular responses and responses to motion in depth (Hoffmann, 1983; Grasse, 1994).

Unlike the turtle, AOS cells in the rabbit have high spontaneous spike rates and direction tuning showing noncolinear excitatory and inhibitory components (Simpson et al., 1988; Soodak and Simpson, 1988), indicating that AOS cells receive visual input that prefers substantially different preferred directions in spatially 
segregated retinal areas. Using intracellular AOS recordings in the reduced turtle brain, no such inputs were observed. The EPSP responses evoked by local moving patterns had similar preferred directions (average difference of $\sim 13^{\circ}$ ) independent of their position within the receptive field. It is possible that BON cells in the intact animal show more complex receptive field properties, or that several of the $\sim 30^{\circ}$ receptive fields in the BON contribute to neurons elsewhere in the brain that encode the optic flow of the full binocular visual field in a three-dimensional visual environment.

In conclusion, this report has directly demonstrated the convergence of retinal ganglion cells onto single neurons of the accessory optic system and supports the role of retinal DS processing in the optokinetic system. Modulation of these excitatory inputs by stimulation outside of the receptive field or by indirect visual inputs mediated through other brain structures are currently under investigation. However, DS retinal cells have been described in most vertebrates, and they may still form the essential sensory path to the accessory optic system.

\section{REFERENCES}

Amamoto DY, Ariel M (1993) A low-cost VGA-based visual stimulus generation and control system. J Neurosci Methods 46:147-157.

Amthor FR, Oyster CW (1995) Spatial organization of retinal information about the direction of image motion. Proc Natl Acad Sci USA 92:4002-4005.

Ariel M (1989) Analysis of vertebrate eye movements following intravitreal drug injections. III. Spontaneous nystagmus is modulated by the GABAa receptor. J Neurophysiol 62:469-479.

Ariel M (1990) Independent eye movements in the turtle. Vis Neurosci 5:29-41.

Baldo MV, Britto LR (1990) Accessory optic-pretectal interactions in the pigeon. Braz J Med Biol Res 23:1037-1040.

Bowling DB (1980) Light responses of ganglion cells in the retina of the turtle. J Physiol (Lond) 299:173-196.

Burns S, Wallman J (1981) Relation of single unit properties to the oculomotor function of the nucleus of the basal optic root (accessory optic system) in chickens. Exp Brain Res 42:171-180.

Fan TX, Rosenberg AF, Ariel M (1993) Visual-response properties of units in the turtle cerebellar granular layer in vitro. J Neurophysiol 69:1314-1322.

Fan TX, Weber AE, Pickard GE, Faber KM, Ariel M (1995) Visual responses and connectivity in the turtle pretectum. J Neurophysiol 73:2507-2521.

Fourment A, Hirsch JC, Marc ME, Guidet C (1984) Modulation of postsynaptic activities of thalamic lateral geniculate neurons by spontaneous changes in number of retinal inputs in chronic cats. 1. Inputoutput relations. Neuroscience 12:453-464.

Granda AM, Fulbrook JE (1989) Classification of turtle retinal ganglion cells. J Neurophysiol 62:723-737.

Grasse KL (1994) Positional disparity sensitivity of neurons in the cat accessory optic system. Vision Res 34:1673-1689.
Grasse KL, Cynader M, Douglas R (1984) Alterations in response properties in the lateral and dorsal terminal nuclei of the cat accessory optic system following visual cortex lesions. Exp Brain Res 55:69-80.

Hamassaki DE, Gasparotto OC, Nogueira MI, Britto LR (1988) Telencephalic and pretectal modulation of the directional selectivity of accessory optic neurons in the pigeon. Braz $\mathrm{J}$ Med Biol Res 21:649-652.

Hoffmann KP (1983) Effects of early monocular deprivation on visual input to cat nucleus of the optic tract. Exp Brain Res 51:236-246.

Kogo N, Ariel M (1996) Direction-sensitive ipsp input to the accessory optic system of turtle. Soc Neurosci Abstr 22:639.

Kogo N, Ariel M (1997) Membrane properties and monosynaptic retinal excitation of neurons in the turtle accessory optic system. J Neurophysiol 78:614-627.

Krapp HG, Hengstenberg R (1996) Estimation of self-motion by optic flow processing in single visual interneurons. Nature 384:463-466.

Natal CL, Britto LR (1987) The pretectal nucleus of the optic tract modulates the direction selectivity of accessory optic neurons in rats. Brain Res 419:320-323.

Natal CL, Britto LR (1988) The rat accessory optic system: effects of cortical lesions on the directional selectivity of units within the medial terminal nucleus. Neurosci Lett 91:154-159.

Northmore DP, Granda AM (1991) Ocular dimensions and schematic eyes of freshwater and sea turtles. Vis Neurosci 7:627-635.

Oyster CW, Takahashi ES, Collewijn H (1972) Direction-selective retinal ganglion cells and control of optokinetic nystagmus in the rabbit. Vision Res 12:183-193.

Oyster CW, Amthor FR, Takahashi ES (1993) Dendritic architecture of ON-OFF direction-selective ganglion cells in the rabbit retina. Vision Res 33:579-608.

Peterson EH, Ulinski PS (1979) Quantitative studies of retinal ganglion cells in a turtle, Pseudemys scripta elegans. I. Number and distribution of ganglion cells. J Comp Neurol 186:17-42.

Rosenberg AF, Ariel M (1990) Visual-response properties of neurons in turtle basal optic nucleus in vitro. J Neurophysiol 63:1033-1045.

Rosenberg AF, Ariel M (1991) Electrophysiological evidence for a direct projection of direction-sensitive retinal ganglion cells to the turtle's accessory optic system. J Neurophysiol 65:1022-1033.

Rosenberg AF, Ariel M (1996) A model for optokinetic eye movements in turtles that incorporates properties of retinal slip neurons. Vis Neurosci 13:375-383.

Simpson JI, Leonard CS, Soodak RE (1988) The accessory optic system of rabbit. II. Spatial organization of direction selectivity. J Neurophysiol 60:2055-2072.

Soodak RE, Simpson JI (1988) The accessory optic system of rabbit. I. Basic visual response properties. J Neurophysiol 60:2037-2054.

Vaney DI (1994) Territorial organization of direction-selective ganglion cells in rabbit retina. J Neurosci 14:6301-6316.

Wylie DR, Frost BJ (1990) Binocular neurons in the nucleus of the basal optic root (nBOR) of the pigeon are selective for either translational or rotational visual flow. Vis Neurosci 5:489-495.

Zhang D, Eldred WD (1994) Anatomical characterization of retinal ganglion cells that project to the nucleus of the basal optic root in the turtle (Pseudemys scripta elegans). Neuroscience 61:707-718. 